bution to the observed value of $\Delta \mathrm{T} / \mathrm{T}$ at comparatively large $\mathrm{W}$ $\left(\mathrm{W} \geqslant 0.24-0.31\right.$ when $\left.\Omega \mathrm{h}^{2}=1-0.1\right)$ which are of most importance in the whirl cosmogony.* Comparison with the observed upper limit $\Delta \mathrm{T} / \mathrm{T} \leqslant 10^{-4}$ shows that the whirl theory in its present state is invalid unless $\Omega \mathrm{h}^{2} \geqslant 0.3-0.4$.

We conclude that both primaeval whirls or "white noise" adiabatic density perturbations responsible for galaxy formation may be compatible with the available upper limits to $\Delta \mathrm{T} / \mathrm{T}$ only in a dense enough Universe $\left(\Omega h^{2} \geqslant 0.3-0.4\right)$.

\title{
REFERENCES
}

Anile, A.M., Danese, L., De Zotti, G. and Motta, S., 1976. Astrophys.J., Chibisov, G.V., 1972. Astron. Zh., 49, 74.

Chibisov, G.V. and Ozernoy, L.M., 1969. Astrophys. Lttters, 3, 189. Kurskov, A.A. and Ozernoy, L.M., 1977a. Astrophys. Sp. Sci. (submitted). Kurskov, A.A. and Ozernoy, L.M., 1977b. Astrophys. Sp. Sci. (submitted). Zeldovich, Ya. B. and Sunyaev, R.A., 1972. Astrophys. Sp. Sci., $\underline{7}, 3$.

*It should be noted that the value of $\Delta \mathrm{T} / \mathrm{T}$ from the last scattering calculated by Anile et al. (1976) and used by Parijskij (this meeting) was overestimated by about one order of magnitude (Kurskov and Ozernoy 1977b).

\section{RECENT ADVANCES IN MICROWAVE COSMOLOGY}

\section{Paul Boynton}

In summary, this brief communication outlined first the recent measurement of large-scale anisotropy of the relic radiation by the Berkeley group of Smoot, Gorenstein and Muller (1977). The customary peculiar velocity interpretation of the dipole term gives $v=390 \pm 60$ $\mathrm{km} / \mathrm{sec}$ in the direction of $\alpha=11.0 \pm 0.5$ and $\delta=6^{\circ} \pm 10^{\circ}$. These values differ from the preliminary results of Corey and Wilkinson (1976) by less than twice their reported errors.

Secondly, the latest observations by G. Lake and R. B. Partridge (1977a, 1977b) of clusters of galaxies seeking evidence for inverse Compton "cooling" of the microwave background were reviewed. Significant effect was indicated in each of the richness class 4 clusters observed:

\begin{tabular}{cc} 
Abe11 Cluster & $\Delta \mathrm{T} \pm 1 \sigma$ \\
\hline 1689 & $-1.06 \pm 0.46 \mathrm{mK}$ \\
2125 & $-3.10 \pm 0.34 \mathrm{mK}$ \\
2218 & $-2.65 \pm 0.23 \mathrm{mK}$ \\
\hline
\end{tabular}


The remaining 8 clusters (richness class 2 and below) showed no temperature deviations beyond $\pm 0.4 \mathrm{mK}( \pm 2 \sigma)$. All measurements were made at $9 \mathrm{~mm}$ with a resolution of $\sim 4^{\prime}$. Some of the values quoted above have been "updated" since the symposium.

\section{REFERENCES}

Corey, B.E., and Wilkinson, D.T., 1976. Bulletin of the AAS, 8, 351 . Lake, G., and Partridge, R.B., 1977a. Nature (to be published). Lake, G., and Partridge, R.G., 1977b. In preparation. Smoot, G.F., Gorenstein, M.U., and Muller, R.A., 1977. Ap. J. Letters (to be published).

\section{DISCUSSION}

Silk: There seemed to be a positive deflection in the direction of Abe11 426 .

Boynton: Yes. This is the Perseus cluster in which there is known to be an intense discrete radio source at centimetre wavelengths.

Parijskij: I mentioned on Tuesday that the new result by Partridge on the Sunyaev-Zeldovich effect in the Coma cluster does not contradict our result in the sense that they do not reject the possibility that the effect exists. The numbers quoted by Partridge and by us do disagree and new observations should be made with special attention to the problem of discrete sources.

At the same time, it seems to me that in the Coma cluster the effect is rather small compared with ones expectation from the intensity and spectrum of the $\mathrm{X}$-ray emission. The mass of gas obtained from observations of $\Delta T / T$ seems to be less than that from the intensity of the $\mathrm{X}$-ray emission. This may be due to clumpiness in the gas. It may we 11 be that in the future the best way to find this out is to take the temperature from the $\mathrm{X}$-ray observations and compute the mass from the Sunyaev-Zeldovich effect.

Boynton: Let me just comment that while your result may be consistent with Partidge's result at your $3 \sigma$ level, his is not consistent with yours at his $3 \sigma$ level. 